\title{
Research on Creative Clothing Design Based on 3D Printing Technology
}

\author{
Li Zhang ${ }^{1,2}$ \\ ${ }^{1}$ Jiangxi center for Modern Apparel Engineering and Technology, China \\ ${ }^{2}$ Jiangxi Institute of Fashion Technology, China \\ lizhang_I_z@sina.com
}

Keywords: 3D printing technology; Creative clothing design; Strategy.

\begin{abstract}
A series of new technologies emerge unceasingly with the progressive development of human society. Novel manufacturing technology represented by 3D has begun to change the mode of traditional manufacturing industry. Auxiliary function at certain degree is also available especially in the aspect of clothing design. Therefore, stereo modeling which can not be completed in traditional clothing design can be realized. In the research of the paper, the author discusses development condition of current 3D printing technology, and combines creative clothing design for in-depth exploration. Corresponding opinions and suggestions are proposed.
\end{abstract}

\section{Introduction}

Many parties pay attention to 3D printing technology since its birth. It can assist clothing manufacturing industry to save cost to a large extent. Therefore, the technology has great prospect for development. Meanwhile, the application of 3D printing technology in the field of clothing has achieved good results, thereby enriching the clothing industry.

\section{Overview and Development Trend of 3D Printing Technology}

Concept of 3D Printing Technology. 3D printing technology does not belong to a sudden breakthrough technology. It has occurred in horizon of people in the last century. It realized real prosperity since this century. American Association of Materials and Test defines material increase manufacturing technology as follows: layer-by-layer staking mode, on the contrary to reduction manufacturing technology, is adopted for producing objects based on 3D model data. Materials are overlapped layer by layer through computer control generally. Finally, 3D model on the computer is changed into a three-dimensional object, and it is a leading technology of developing from mass production mode to personalized manufacturing mode. 3D printing technology brings greater influence to all industries after being developed till present and its influence is self-evident especially in industrial product and medical technology fields.

Advantage of 3D Printing Technology. 3D printing technology only belongs to a leading technology from the aspect of nature, which can promote transformation from mass production mode to personalized manufacturing mode. The manufacturing industry is effectively driven to realize low cost and high efficiency under such mode transformation. 3D printing technology belongs to a forming mode which is increased layer by layer from the aspect of core ideas. The use mode of 3D printing technology is objectively determined by such a mode. It only needs to focus on the existence of object in spatial position rather than material processing mode at the traditional level. Meanwhile, 3D printing technology can effectively improve the utilization efficiency of resources with more evident environmental protection effect.

Development Condition of 3D Printing Technology. Firstly, 3D printing technology belongs to an ideal mode which can realize synchronous molding of various materials, and make up for the inadequacy of traditional manufacturing production. In fact, because there are various limitations of 
objective conditions in the traditional manufacturing industry, the cost increased due to the process difficulty is far higher than the benefits brought by the technology. Therefore, more than two materials cannot be manufactured synchronously; however the situation is changed due to emergence of $3 \mathrm{D}$ printing technology.

Secondly, metal printing at low cost is achieved. The printing mode can broaden the practicability of manufacturing industry. In addition, 3D printing technology can be used for designing different styles according to different parts on the basis of alloy composition proportion design.

\section{Overview of Modern Creative Clothing}

Design Method of Creative Clothing. The innovation design of modern clothing can be mainly explored in the aspects of structure, detail, color etc. Application of technology in clothing innovation has become a major trend with development of economy, science and technology. Combination of design perception aesthetics and rational aesthetics can be greatly embodied. 'Novelty' is the most important embodiment of creative clothing. A variety of art forms are comprehensively used for creating clothing with unique meanings. There are more novel ideas in the aspects of clothing composition and design. Creative clothing is different from ordinary clothing. Ordinary clothing only focuses on color and style, and creative clothing pay much attention to texture change of clothing fabric. Therefore, fabrics with innovative design and texture special transformation are important elements of creative clothing (as shown in Fig .1).
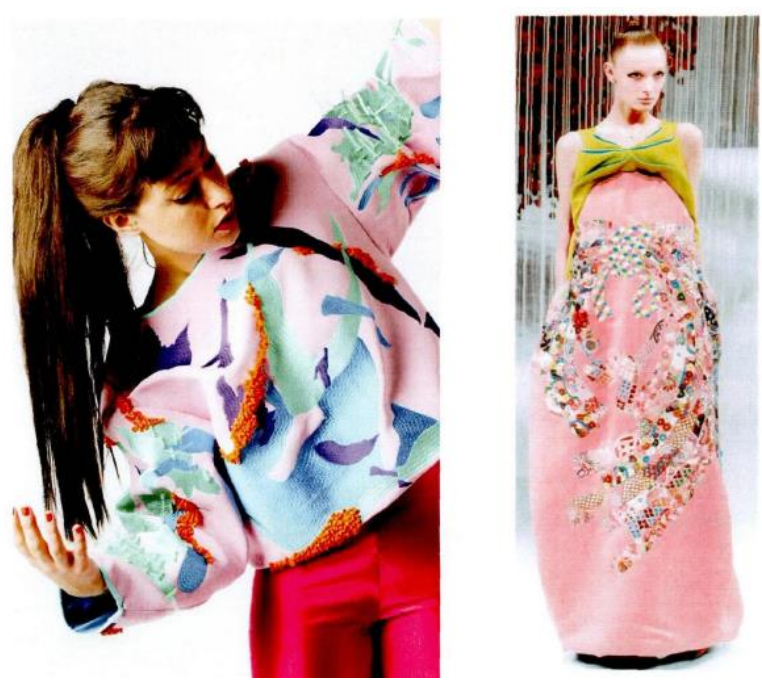

Figure 1. Material innovation design

Design Characteristics of Creative Clothing. Creative clothing key lies in that it can be different from ordinary clothing. It can fully reveal out new characteristics in the clothing field. Creative clothing has general performance characteristics of non-clothing performance, fuzziness and none center according to the existing practice experience. Non-clothing performance mainly refers that the clothing is made of paper, plastic, wood, CD and other materials, which are applied in creative clothing design (Fig. 2). Such a design mode can bring visual enjoyment to human beings. Fuzziness refers that diverse elements are gathered together, and various techniques are flexibly used. Non-center refers that the whole work is free from prominent boundaries in the whole clothing design process. Accessory is applied in creation as a unique character. Its significance is larger than itself (as shown in Fig. 3). 

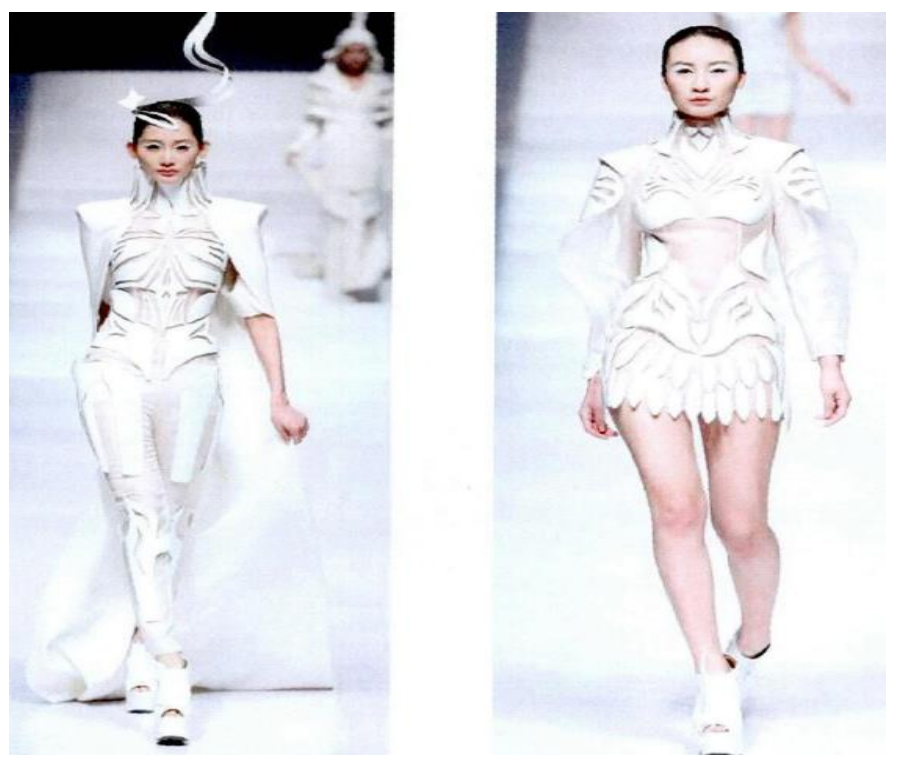

Figure 2. Application of plastic cut-parts in creative clothing

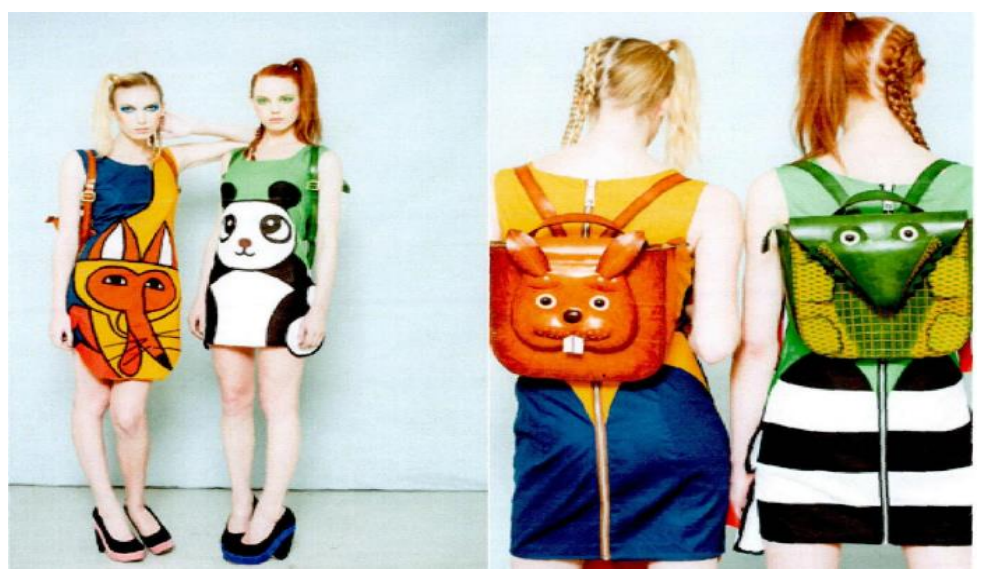

Figure 3. Non-center of creative clothing

Development Trend of Creative Clothing. Creative clothing always exists in a unique mode of design language since its birth. It has stronger innovation and implication. Creative clothing accounts for a lower proportion in the market due to technical limitations and backward consumer concept from the perspective of market. However, we can constantly search new styles due to existence of creative clothing, thereby paving new materials and studying new process, which plays an important role for further expanding marketization. Characteristic and excellent creative clothing can bring new inspiration to consumers from another perspective, thereby further leading customers for consumption, and improving the value of the whole society in the field of clothing. Since high technology products in the society should be adopted as reference for the creative clothing itself, therefore creative clothing trend also can lead development and perfection of clothing industry in the future from the perspective of industrialization. Therefore, 3D printing technology can be combined with creative clothing for largely exploring elements in creative clothing. Clothing fashion culture is popularized for better displaying creative clothing design in modern features. 


\section{Combination of 3D Printing Technology and Creative Fashion}

Design Category Clothing Material. Clothing materials mainly include cotton, hemp, silk, wool, chemical fiber products and other categories traditionally, and they are woven into yarn through spinning technology. Then, they can form fabric through knitting or weaving structure. In the production process, corresponding color dying or printing technology can be integrated to form colorful patterns. Yarns with different thicknesses or structures can be combined for realizing flexibility and change of clothing fabric.

Diversified clothing production modes are presented in front of people with the emergence and development of 3D printing technology. Though current 3D printing technology is limited in the aspect of material application, clothing of textile fabric cannot be printed. However, application of 3D technology in the aspect of plastic materials has reached certain level, including ABS plastic and PLA plastic. Creative clothing materials under 3D printing technology reflect postmodern style. The structures mostly belong to ring structures. The expressed con notations are mostly fit with ideas of designers rather than clothing which is worn by people in daily life. It is worth mentioning that since all current 3D printing clothing is made of plastic, designers always show plastic design in the mode of ring shape or flake shape. They are mutually combined. If certain comfort should be equipped for the clothing, clothing materials should be considered well, thereby reflecting certain scientific nature and rationality.

Clothing Color. Color is an indispensable important element in the field of clothing design, which not only produces direct impact on people's vision, but also leads the trend and style of clothing. Similarly, clothing color is also an important aspect in 3D printing technology. Accurate expression of each color in $3 \mathrm{D}$ printing has been more and more emphasized for long term.

Current existing situation shows that people mainly apply the following concentrated solutions in the aspect of treating colors of 3D printed objects: (1) hydrology transfer printing method; So-called transfer printing is printing in the traditional sense. Transparent film should be prepared in the process of utilizing the method; patterns should be printed on the film. Then, the patterns can be arranged on water, and auxiliary reagents should be added. After the above steps are completed, colored objects should be placed in reagent, films should be arranged around the object, therefore color can be printed on the surface of the object, and this is so-called hydrology transfer printing method. The aspect has been widely applied currently, good effect can be brought, but the method also has certain defects. Since the film can show gradual expansion condition after being arranged in water, thereby the method has low accuracy. (2) Powder integration or pavement of extruded plastic; simplicity is one significant feature under such printing mode. Colors are controlled more singly, and materials in different colors can be integrated through many nozzles, thereby realizing color change. Though the mode can be operated more simply, it is not widely applied in the field of clothing design due to color limitation. (3) Pixel inkjet method; the method refers to innovation on the basis of traditional 2D. Main operation procedures are shown as follows: ink jetting is utilized for paving on the surface of objects. Since the ink drops can be cured by the ultraviolet rays, which can become solid, patterns and color can be stored. The method has high requirements on accuracy and technology. Huge data calculation is also involved during use, and therefore there are limitation factors during use of the technology.

Clothing style. Former description shows that 3D printing technology has a prominent advantage in creative clothing design that production links can be observed, manpower, material and financial resources can be saved in traditional clothing design. Comprehensive automation can be realized. Even when the technology is developed to certain extent, each person can design our desired clothing by the aid of the technology. All styles required in current creative clothing design can form a huge database with popularity of digital technology. The styles can be flexibly changed through modifying contents in the database, thereby improving the convenience of the operation. 
3D Printing and Creative Clothing Design Process. 3D printing is applied in the whole process of creative clothing design, which can objectively promote creative clothing design to realize unification. In addition, resource sharing can be realized by all links and resources in the design. Specifically, mold or fixture should not be additionally used under 3D technology. Physical shape and structures are not limited, combination change at all levels and all dimensions can be realized. Therefore, there is no direct link between manufacturing cost and batch. Firstly, a lot of cost in the traditional manufacturing process can be saved by 3D technology. Secondly, process flexibility also can be increased. For example, CONTINUUM FASHION AND SHAPEW AYS Co., Ltd. issued the first 3D printing bikini all over the world on June 6, 2011 (as shown in Fig. 4). In the 3D printing creative clothing, designers can organically combine 'string', 'large and small dots' and other design elements together. The clothing designed is coordinated with human body line proportion. All limitations in traditional design are changed. A brand-new mode is adopted for cutting cups.

In addition, 3D printing technology is applied in creative clothing design. Clothing design concept can be extended. More complicated data contained or involved in the clothing are operated on the basis of existing technology. Our familiar visual and interactive operations are contained. On the basis, designers can generate clothing three-dimensional physical goods through rapid solidification laser material preparation and forming technology.

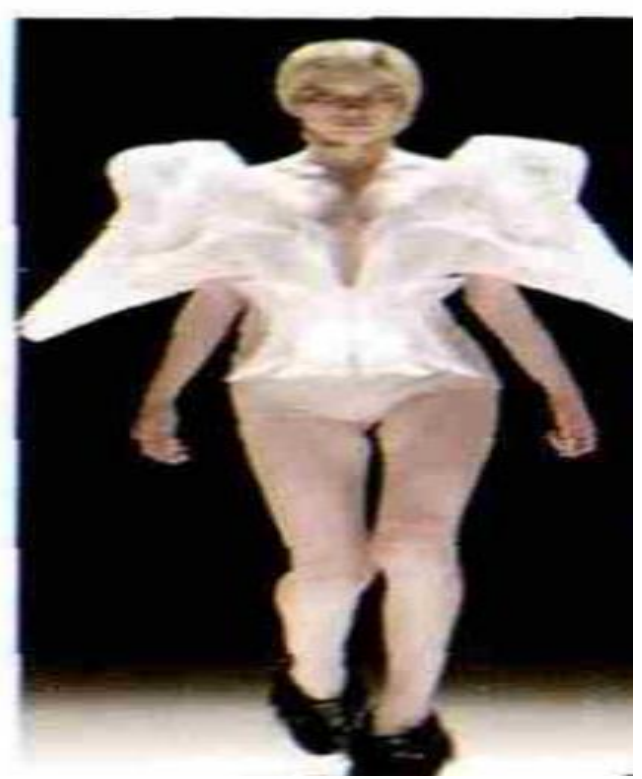

Figure 4. 3D printing bikini

3D Printing and Creative Clothing Industry Mode. 3D printing technology brings certain technology to development of creative clothing industry mode. Computer technology can play certain value in solid object scanning through 3D scanner. It makes up for communication shackle between clients and designers in traditional mode. Therefore, object model and relational database can be generally mastered through corresponding mapping theory, thereby finally completing physical implementation. Nike Company displays the first pair of 3D printing technology sport shoe soles designed for American footballers outwards during new talent training on February 23, 2013 (as shown in Fig. 5). The shoes have lighter weight, which also can show the duration of the most original driving state of athletes, and good practical effect is achieved. 


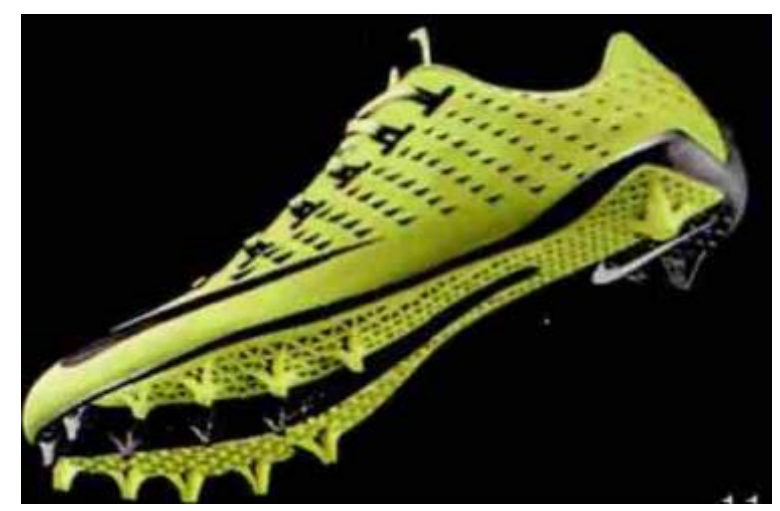

Figure 5. 3D printing technology sports shoes of Nike Company

\section{Prospect Outlook of 3D Printing Creative Clothing}

In the future, services of $3 \mathrm{D}$ clothing products can be gradually perfected with the further development and innovation of 3D printing technology, improvement and updating of relevant materials. It can gradually meet dressing demand of different consumption groups. Since there is certain correlation between 3D printing technology and many disciplines, interdisciplinary knowledge can be applied in clothing design. It will be a great trend in creative clothing design in the future. It is provided with innovative mode of thinking and subverts stereo modeling consciousness on the basis of inheriting tradition, thereby controlling the latest 3D printing technology comprehensively.

\section{Conclusion}

We can predict that future 3D printing technology will achieve more considerable development prospects based on current development situation of science and technology. Designers do not need to limit our sight to clothing cutting mode, and they should widen design thinking and try to show own design thinking on the computer. Creative clothing design can be improved to a brand-new level only through suitable fabric and flexible technology application ability, thereby driving sustainable development in the field.

\section{References}

[1] Zhou Li, Shen Yue, Zhang Longlin. Analysis on clothing design based on 3D printing technology Decoration, 2014, 05: 88-89.

[2] Xiao Xiao. Application of 3D printing technology in personalized creative design. Design Art Studies, 2015, 01: 70-73.

[3] Song Jia, Yuwen Taman. On application prospect of 3D printing technology in clothing design. Small and Medium-sized Enterprise Management and Science Technology, (middle-month journal), 2015, 10: 201.

[4] An Ni, Wang Jia. Discussion of influence of 3D printing technology on clothing customization industry. Shanghai Textile Technology, 2015, 05: 1-4.

[5] Creation of the world and construction of everything- 3D printing technology for changing the world. Invention and Innovation (integrated science and technology), 2011, 11: 4-9.

[6] Zhou Lulu. Development trend of 3D printing technology instead of digital printing in future clothing design. Blest Garden 2015, 06: 87-89. 
[7] An Ni, Li Qiju, Peng Fanke. Application of 3D printing technology in the production of women's corsets. Journal of Textile, 2014, 04:154-159.

[8] Liu Shuying, Elizabeth Mandela. 3D technology trend and development trend of textile and garment industry. China Fiber Inspection, 2015, 16:60-63.

[9] Wu Chaogang. Application of 3D printing technology in the modern clothing. Art Science and Technology, 2015, 01: 11+127+168.

[10]Zhang Mengzhu. Analysis on clothing design development trend based on 3D printing. Art Science and Technology, 2015, 04: 97. 